\title{
Somatoform Disorders in Parkinson's Disease and Dementia with Lewy Bodies Evidence Underlying Psychotic Traits
}

\author{
Marco Onofrja ${ }^{\text {- Astrid Thomas }}{ }^{\text {- Laura Bonanni }}$. \\ Massimo di Giannantoniob $\cdot$ Francesco Gambib $\cdot$ Gianna Sepede ${ }^{b}$ \\ aNeurology Clinics and bsychiatry Clinics, Department of Neuroscience and Imaging and Aging Research \\ Center, Ce.S.I., 'Gabriele d'Annunzio' University Foundation, University G. d'Annunzio of Chieti-Pescara, \\ Chieti, Italy
}

\begin{abstract}
Somatoform disorders have only recently been described in Parkinson's disease (PD) and dementia with Lewy bodies (DLB). Of 1,210 patients with neurodegenerative diseases referred to our institutions from 1999, 488 were diagnosed with PD, 415 with Alzheimer's disease, 162 with DLB, 48 with progressive supranuclear palsy, 48 with multiple system atrophy, and 49 with frontotemporal dementia. Rates of somatoform disorder were considerably higher in DLB (18\%) and PD (7.5\%) than in any other group (0-2\%). Somatoform disorders in PD and DLB were characterized by motor and non-motor patterns with bizarre presentations, and were often accompanied by catatonic signs (41\%). In 77\%, they preceded PD motor signs by 6-120 months, and in $86 \%$, they were recurrent at follow-up. In $91 \%$, there was preceding or concomitant hypochondriasis. Global cognitive decline was greater in PD with somatoform disorder than without $(p<0.001)$, comparable to that observed in the DLB group. The phenomenology suggests a blurred boundary between somatoform disorder and psychosis (somatic delusions). The presence of somatoform disorder may identify a subgroup of PD patients with distinctive clinical symptoms, including catatonic features and a poor cognitive outcome.

Copyright $\odot 2012$ S. Karger AG, Basel
\end{abstract}

Depression, apathy, and impulse control disorder with its variants, including L-dopa dysregulation syndrome, have a defined status among psychiatric complications of Parkinson's disease (PD), and are discussed in detail in earlier chapters [1]. New evidence suggests that the spectrum of psychiatric symptoms in PD and related diseases may include yet inadequately described disorders. 
A recent survey in 20,140 patients hospitalized in Sweden for PD found that prior hospitalization for schizophrenia, mood disorders, neuroticism and depression increased the risk for the development of subsequent PD. Even taking into account the possible effect of neuroleptic exposure, the study suggests that the relation of mental disorders with parkinsonism is more complex than commonly believed [2]. Psychosis in PD is studied far less often than depression, apathy or impulse control disorder. In many studies, psychosis is used as a synonym for hallucinations, with the latter being considered a core element of dementia with Lewy bodies (DLB) and, less consistently, PD with dementia [3].

In recent years, we have focused our attention on the broad spectrum of somatoform disorders in PD and DLB patients, ranging from the DSM IV-TR somatoform disorder category [4] with its variants to related disorders characterized by bizarre and delusional somatic complaints.

Several observations have attracted our interest in somatoform disorder in PD and DLB: (1) somatoform disorder might precede the onset of neurodegenerative disorders $[5,6]$. (2) Conversion type somatoform disorders are described anecdotally in patients who develop PD [5, 7-9]. (3) Alexithymia (inability to distinguish feelings from sensation of emotional arousal) is increased in PD [10]. (4) Babinski's definition of hysteria (= somatoform disorder) was that it consists of an 'illness that could be induced by suggestion and abolished by persuasion' [11]. Suggestibility is also a feature in PD, as shown by the magnitude of the placebo effect, which may induce a reduction in motor symptom severity up to $30 \%$ [12], confounding pharmacological studies. (5) Historically, a variety of motor or non-motor features different from the well-known postural, gait and motor symptoms have been reported in untreated PD patients. These features consist of abnormal bizarre gymnastic postures, 'poses gymniques', or of somatic complaints or unclassified gait abnormalities, which were categorized as overlapping with hysteria or catatonia. Some of these features were attributed to patients affected by parkinsonism due to encephalitis lethargica, but in others encephalitis was excluded as a cause [13].

\section{A Prospective Cohort Study in Somatoform Disorder in Lewy Body Disease}

In order to clarify the relevance of somatoform disorder in parkinsonism, we designed a prospective cohort study comparing patients with somatoform disorder preceding or concomitant with the onset of motor PD symptoms with randomly selected PD patients without somatoform disorder. Similarly, we compared DLB patients with somatoform disorder with those without somatoform disorder. These groups were followed up clinically and with different laboratory assessments for at least 4 years in order to understand whether the presence of somatoform disorder affected motor and cognitive outcomes, and we report some of the findings [14] here and give updates of our more recent cohort data. 
Prior to our study, somatoform disorder had been described as a prodromal symptom of Alzheimer's disease in two studies performed in a resident population of nuns $[6,14]$. Elderly subjects who presented with somatoform disorder (described as hysteria in these papers) in the course of their life, were shown to be more prone than others to develop dementia with advancing age.

At the time of the publication of our study [15], a review described somatoform disorders among the non-motor psychiatric manifestations of parkinsonism and PD [16], and a study based on a neuropsychiatry scale reported a $40 \%$ prevalence of somatizations in PD [17].

\section{Definition: Somatoform Disorder}

The definition of somatoform disorder in DSM-IV-TR [4] relates to a general category including seven, partly disparate disorders: (1) somatization disorder, (2) somatic body dysmorphic disorder, (3) delusional disorder somatic type, (4) undifferentiated somatoform disorder, (5) conversion disorder, (6) pain disorder, and (7) hypochondriasis. Each has a different frequency in the general population, varying from $0.2-2 \%$ for somatization or conversion disorder to $3-13 \%$ for hypochondriasis. Despite this heterogeneity, the core entity defined by DSM-IV-TR somatoform disorder is a somatoform complaint that is incongruent with rules of anatomy and physiology in the absence of any specific evidence of organic illness in medical and laboratory assessments.

\section{Prevalence of Somatoform Disorder in Patients with Neurodegenerative Disorders}

In the 10 years from 1999, 1,572 new patients were evaluated in our Movement Disorder and Memory Clinic. Over at least 2 years of follow-up, 943 patients were classified under neurodegenerative disorders. Four hundred and eighty-eight were diagnosed with PD (or genetic parkinsonism), 415 with Alzheimer's disease, 162 with DLB, 48 with progressive supranuclear palsy, 48 with multiple systems atrophy, and 49 with frontotemporal dementia. Seventy-four patients received a diagnosis of concomitant somatoform disorder. Among them, 9 patients were affected by psychogenic movement disorders or catatonia, 7 patients were affected by Alzheimer's disease, and one had progressive supranuclear palsy.

A significant proportion of patients with somatoform disorder were affected by Lewy body disease; 29 PD patients had somatoform disorder at the time of PD diagnosis, and in 7 somatoform disorder symptoms had been observed in the 6 preceding years. Definite somatoform disorder was also found in 29 DLB patients, and in the majority (93\%) of these hospital notes gave a history of incongruent somatoform complaints. All were reported by caregivers and general practitioners to have had 
hypochondriasis. Somatoform disorder had preceded DLB by 12 years in one patient, and by $6-2$ years in the other 14 patients, with the remainder having somatoform disorder in parallel with their diagnosis of DLB.

\section{Follow-Up of Patients with Lewy Body Disease and Somatoform Disorder}

In our 9-year longitudinal study, MMSE and 'frontal' battery test scores decreased significantly in PD patients with somatoform disorder, compared with patients without somatoform disorder. However, the progression of decline was similar in all DLB patients regardless of whether they had somatoform disorder or not. At the end of the 9-year follow-up period, all patients with PD-somatoform disorder, DLB-somatoform disorder and DLB without somatoform disorder were institutionalized, whereas none of the PD patients without somatoform disorder required institutional care.

\section{Clinical Phenomenology and Overlap of Psychotic Symptoms}

We identified three characteristics features in PD-somatoform disorder patients:

First, a temporal pattern of occurrence or recurrences of somatoform disorder was observed in all patients, with somatoform disorder episodes appearing in clusters lasting from 2 weeks to 3 months; only in 2 patients did the disorder last longer than a year. These episodes had characteristics of DSM-IV-TR somatization disorder, or of conversion disorder, or of intense somatic type delusional disorder often with bizarre or Cotard type contents, indicating the need for further classification, and of catatonia.

Second, in $73 \%$ of patients there was denial of the diagnosis of parkinsonism and occurrence of marked side effects with initial dopaminergic drug exposure. Adverse responses included intolerable gastric pain, nausea, burping and retching despite concomitant treatment with $\mathrm{H} 2$ antagonists, ondansetron or granisetron, domperidone, globus pharyngeus after ingesting pills, but not food or liquids, confusion, sensation of empty headedness, dizziness with normal orthostatic blood pressure, hypersomnolence or insomnia.

Third, a clear history of hypochondria occurred in $92 \%$ of subjects. The essential feature was preoccupation and centrality of concerns about disease, doctor shopping, frustration and anger in the doctor-patient relationship, which was consistently reported by relatives and general practitioners in $91 \%$ patients and corroborated by hospital records documenting several admissions for minor disorders in $82 \%$ patients.

Somatoform disorder symptoms could be motor or non-motor. Yet in all patients apart from examples of simple motor and non-motor patterns, including hemiparesis, paraparesis, anesthesia, hypoesthesia, with classic conversion signs including 
distractibility and Hoover sign (consisting of absent pressure on contralateral talon when attempting to lift, lying in bed, the pseudo-paretic lower limb), the outstanding symptoms often presented with bizarre patterns.

\section{Conclusions}

We identified symptoms of somatoform disorder in a clinically significant proportion of PD patients (7.5\% in the updated cohort data) and in an even higher percentage of DLB patients (19\%).

Symptoms observed in our patients included those described under the different subgroupings of DSM-IV-TR: typical of conversion disorders (like bent knee and tiptoeing, posturing, hemiparesis or paraparesis, psychogenic movement disorders), of somatization disorders (like unexplained and multi-localized pain, anesthesia, hypesthesia), and bizarre somatoform delusions (bizarre delusions of progressive deformation of body parts). These were observed concomitantly with the onset of PD motor signs or during $\mathrm{PD}$ progression, and were recurrent during the course of PD. During recurrences, somatoform symptoms appeared with different patterns. Hypochondria, listed by DSM-IV-TR among somatoform disorders, was also a consistent presentation in the medical history of the majority of patients (91\%), leading to hospitalization years before motor PD symptoms had appeared. When PD was diagnosed, denial and resistance to the PD diagnosis was consistently observed in patients with hypochondriasis. While counterintuitive, this feature may be construed taking into account psychodynamic hypotheses of manipulative anger [18].

In $41 \%$ of PD and DLB patients with somatoform disorder, catatonic symptoms were observed including negativism, immobility, abnormal posturing, waxy flexibility and stereotypies. This frequency suggests that catatonia should be sought specifically in addition to the more global search for the wider variety of somatoform disorder symptoms.

Neurobiological hypotheses suggest that somatoform disorder and catatonia in general are different grades of expression of a same pathological entity, resulting from the dysfunction of frontal lobes-basal ganglia-thalamus interactions [19]. Our study confirms that somatoform disorder and catatonia can appear as a continuum, at least in the group of patients affected by PD or DLB.

Somatoform disorder can appear in parkinsonism with catatonia and somatic delusions, Cotard or Ganser syndrome, which are properly classified as psychotic symptoms. We suggest that the boundary between somatoform disorder and psychosis is blurred in PD and DLB patients. The blurring and overlap between somatoform and psychotic disorders further underlines the need to improve categorization and understanding of types of psychotic symptoms in PD and DLB. The consensus criteria for DLB include delusions among the supportive elements needed for the diagnosis. Our 
previous report [15] suggests that somatic delusions play a dominant role in these diseases and should be sought for in order to support the diagnosis.

The second conclusion briefly presented here is that presence of somatoform disorder is a prognostic predictor for the development of dementia of the DLB type in patients with PD. The progression of PD motor symptoms was similar in PD patients with somatoform disorder without somatization, while the progression of cognitive test scores was different. Somatoform disorder predicted a decline in executive function, and a faster progression to subcortical-frontal dementia. Once a patient with somatoform disorder developed objective PD signs, the progression to dementia or institutionalization could be reliably predicted. Somatoform disorder may precede the occurrence of clinical and imaging evidence of PD by years, but once PD signs have developed the pattern of progression is more similar to DLB than the progression observed in PD without somatoform disorder.

It could be argued that the need for institutionalization could be due to repeat requests for medical care, one of the presenting features of somatoform disorder. However, in our survey recurrences of somatoform disorder diminished as dementia scores increased; thus, it is more likely that the need for institutionalization was due to the presence of psychosis and dementia.

For comparison, our studies included several groups of patients recruited in the same setting and affected by Alzheimer's disease, frontotemporal dementia and progressive supranuclear palsy. The comparison with these patients showed that somatoform disorder, or the evidence of prior hospitalization for somatoform disorder, was significantly lower in patients with these diseases than in PD or DLB patients.

We could not confirm the results of a previous cohort study showing that somatoform disorder predicted the occurrence of dementia of the Alzheimer type $[6,14]$. However, when data for the prior study were collected, recognition of DLB or dementia associated with synucleinopathy was infrequent, and it might be argued that categorization of dementias into Alzheimer's disease was, at the time, excessive.

The low frequency of somatoform disorder in patients with frontotemporal dementia and progressive supranuclear palsy was surprising, as frontotemporal dementia and progressive supranuclear palsy are undoubtedly examples of frontal lobe dementia. This finding suggests that further investigations may be needed on the anatomical pattern of frontal lobe dysfunction and pathway disruption in these three disorders. Neuropsychological tests showed that, at onset, progressive supranuclear palsy and frontotemporal dementia patients had worse scores than PD patients presenting with somatoform disorder. We hypothesize that, in order to express somatization disorder, the frontal lobe should be dysfunctional but partly preserved, while a severe loss of frontal lobe function may abolish the ability of somatization. This hypothesis is consistent with observations that patients who presented with somatization disorders did so before or concomitantly with PD onset or in the following 2 or 3 years, while this clinical pattern disappeared due to dementia in the long-term follow-up. 
A recent neuroimaging study showed that focal hypoactivity in precuneus and supramarginal gyri, when concomitant with hyperactivation of orbitofrontal areas, is linked with the expression of unconscious somatizations [20]. This recent discovery relocates the focus of imaging studies on somatoform disorder and forecasts innovative approaches to these disorders. Parietal areas, including precuneus and supramarginal gyrus, are in fact considered essential in providing integration of internal and extra-personal (visual or sensory polymodal) space. In DLB and PD with dementia patients, imaging studies show specific hypoactivity of parieto-occipital areas [20]. Therefore, the recent study [21] supports the hypothesis suggesting that somatoform disorder should be present and frequent in these diseases.

The prevalence of somatoform disorder matches the prevalence of impulse control disorder (8\% in PD) as reported in the literature [4].

In summary, we suggest that somatoform disorder manifestations identify an important subgroup of PD patients with distinctive clinical features and clinical course in terms of cognitive decline. Further studies of PD-somatoform disorder patients may be useful to link non-motor elements of psychiatric behaviors and cognition in PD with the neural networks that could explain their relationship.

Somatoform disorder is not frequent enough to be considered as a PD pre-motor sign (as REM sleep behavior disorder, loss of olfaction, constipation), but its linkage with PD-dementia suggests that it may be a useful tool to study PD-dementia, and that somatoform disorder might be considered as a potential non-motor precursor of PD-dementia and might be added to the supportive features of DLB for consensusbased diagnoses.

Finally, our study indicates the need for further independent epidemiological assessments to confirm the frequencies, as regional service and cultural factors will modify the expression of somatoform disorders in PD and DLB populations.

\section{References}

1 Wolters EC, van der Werf YD, van den Heuvel OA: Parkinson's disease-related disorders in the impulsive-compulsive spectrum. J Neurol 2008; 255(suppl 5):48-56.

- Li X, Sundquist J, Hwang H, Sundquist K: Impact of psychiatric disorders on Parkinson's disease: a nationwide follow-up study from Sweden. J Neurol 2008;255:31-36.

3 McKeith IG, Dickson DW, Lowe J, Emre M, et al: Diagnosis and management of dementia with Lewy bodies: third report of the DLB Consortium. Neurology 2005;65:1863-1872.

4 American Psychiatric Association: Diagnostic and Statistical Manual of Mental Disorders-Text Revised (DSM-IV-TR), ed 4. Washington, American Psychiatric Publishing, 2000.
5 Crimlisk HL, Bhatia K, Cope H, David A, Marsden CD, Ron MA: Slater revisited: 6 year follow up study of patients with medically unexplained motor symptoms. BMJ 1998;316:582-586.

6 Wilson RS, Evans DA, Bienias JL, Mendes de Leon CF, Schneider JA, Bennett DA: Proneness to psychological distress is associated with risk of Alzheimer's disease. Neurology 2003;61:1479-1485.

7 Lang AE, Koller WC, Fahn S: Psychogenic parkinsonism. Arch Neurol 1995;52:802-810.

-8 Factor SA, Podskalny GD, Molho ES: Psychogenic movement disorders: frequency, clinical profile, and characteristics. J Neurol Neurosurg Psychiatry 1995; 59:406-412.

-9 Saint-Cyr JA, Taylor AE, Nicholson K: Behavior and the basal ganglia. Adv Neurol 1995;65:1-28. 
10 Costa A, Peppe A, Carlesimo GA, Salamone G, Caltagirone C: Neuropsychological correlates of alexithymia in Parkinson's disease. J Int Neuropsychol Soc 2007;13:980-992.

11 Babinski J: Définition de l'hystérie. Rev Neurol 1901;9:1074-1080.

12 Goetz CG, Wuu J, McDermott MP, Adler CH, Fahn S, Freed CR, Hauser RA, Olanow WC, Shoulson I, Tandon PK, Leurgans S: Placebo response in Parkinson's disease: comparisons among 11 trials covering medical and surgical interventions. Mov Disord 2008;23:690-699.

13 Baruk H: Traité de Psychiatrie. Paris, Masson, 1959.

$\checkmark 14$ Wilson RS, Arnold SE, Schneider JA, Kelly JF, Tang Y, Bennett DA: Chronic psychological distress and risk of Alzheimer's disease in old age. Neuroepidemiology 2006;27:143-153.

15 Onofrj M, Bonanni L, Manzoli L, Thomas A: Cohort study on somatoform disorders in Parkinson disease and dementia with Lewy bodies. Neurology 2010;74:1598-1606.

16 Gallagher DA, Lees AJ, Schrag A: What are the most important nonmotor symptoms in patients with Parkinson's disease and are we missing them? Mov Disord 2010;25:2493-2500.
17 Siri C, Cilia R, De Gaspari D, Villa F, Goldwurm S, Marco C, Pezzoli G, Antonini A: Psychiatric symptoms in Parkinson's disease assessed with the SCL90R self-reported questionnaire. Neurol Sci 2010;31: 35-40.

18 Kaplan H, Sadock B: Comprehensive Textbook of Psychiatry, ed 5. Lippincott Williams \& Wilkins, 1989.

19 Vuilleumier P, Chicherio C, Assal F, Schwartz S, Slosman D, Landis T: Functional neuroanatomical correlates of hysterical sensorimotor loss. Brain 2001;124:1077-1090.

20 Lobotesis K, Fenwick JD, Phipps A, Ryman A, Swann A, Ballard C, McKeith IG, O’Brien JT: Occipital hypoperfusion on SPECT in dementia with Lewy bodies but not AD. Neurology 2001;56: 643-649.

21 Leenders K, van Beilen M: Imaging of non organic movement disorders; in 7 th International Congress on Mental Dysfunction \& Other Non-Motor Features in Parkinson's Disease, Barcelona, 2010. 